\title{
Dynamic Cascading Failure Model for Blackout Risk Assessment of Power System With Renewable Energy
}

\author{
Leibao Wang ${ }^{1}, \mathrm{Bo} \mathrm{Hu}^{1, *}$, and Kaigui $\mathrm{Xie}^{1}$ \\ ${ }^{1}$ Chongqing University, The State Key Laboratory of Power Transmission Equipment and System Security, 400044 Chongqing, China
}

\begin{abstract}
To assess the blackout risk of power system with high penetration of renewable, the existing cascading failure models need to be improved for capturing the dynamics and relays of renewable generation. In this paper, a dynamic model of cascading failure considering the utility-scale and distributed renewable energy is proposed. With the solution of dynamic equations for power system, the logics of relays are simulated for components such as transmission lines, conventional generators and renewable generations. The failure interactions among sources, networks, and loads are analyzed more comprehensively. In the proposed model, to capture the impact of renewable energy on the system dynamics, the dynamic equations for the utility-scale renewables are constructed with the second generic generation model of WECC (Western Electricity Coordinating Council), and the interactions among distributed renewables and the transmission system are considered in the amount of net load at buses. And to capture the tolerance of renewables for disturbances, the simulation logic is constructed for the voltage relays and frequency relays of utility-scale renewables and the anti-islanding relay of distributed renewables. The presented model is verified on the IEEE 39-bus system. The results show that renewable energy has a significant influence on the cascading failure risk.
\end{abstract}

\section{INTRODUCTION}

Recent blackouts in South Australia [1] and the United Kingdom [2] indicate that the tripping of the utility-scale and distributed renewable energy may be induced by the voltage disturbances and frequency disturbances in transmission system. The tripping of large-scale renewable energy can cause the redistribution of power flow in transmission system, in which some branches may be overloaded. Moreover, the tripping of renewable energy also worsens the disturbances of frequency and voltage in the grid and increases the risk of system instability. Therefore, to evaluate the reliability of a power system with renewable energy, it is necessary to accurately capture the impact of renewable energy on the blackout risk.

To consider the renewable energy in cascading failures, many cascading failure models studied the impact of the uncertainty of renewable generation on the overloading risk of transmission components. For example, the transmission line overloading risk in cascading failure is assessed using the stochastic power flow to model the fluctuations of renewable energy in Ref [3]. In addition to power flow analysis in steady operation, the dynamic security analysis is also an important factor in risk assessment of cascading failure [4].

In order to capture the dynamics in blackouts, Ref [4] establishes a dynamic simulation model for the traditional power system. However, this model does not consider the impact of renewable energy. Ref [5] proposes a blackout model that considers the dynamics of system frequency and the low voltage ride-through control of wind power. But the frequency model adopted was relatively simple, and this model also does not consider the transient stability and the control system in the electromechanical transient. Besides, the existing cascading failure models do not consider the impact of distributed renewable energy sources. When disturbances occur in the transmission system, the distributed renewable energy may be affected and disconnect from the network, which may worsen the power flow distribution and the transient stability of the grid [6] [7].

The commercial simulation software such as PSASP and PSD-BPA is mainly aimed at analyzing the system dynamics under the given operating mode and the given fault. However, it is difficult to deal with the complicated situation having multiple successive actions of controls and relays in the propagation of cascading failures. To this end, a dynamic model for power system with renewables is proposed in this paper by combining the electromechanical transient model of synchronous generators and the second-generation generic dynamic model by renewable energy of Western Electricity Coordinating Council (WECC). The proposed dynamic model describes the power grid regulation ability after the disturbance occurs. In addition to transmission lines, conventional units, low-voltage / low-frequency load

\footnotetext{
${ }^{*}$ Corresponding author: 1137040351@qq.com
} 
shedding, etc., the relays are also considered for the utility-scale and distributed renewable energy sources.

\section{Dynamic model of traditional power system}

Traditional power systems adopt synchronous generators such as thermal power and hydropower for power generation. To describe the dynamics of the system during cascading failures, the following algebraic differential equations are generally constructed [4]:

$$
\left\{\begin{aligned}
0 & =g(x, y, z) \\
\frac{d x}{d t} & =f(x, y, z) \\
z_{k+1} & =h\left(x, y, z_{k}\right)
\end{aligned}\right.
$$

The algebraic equations $g(\mathrm{x}, \mathrm{y}, \mathrm{z})$ in (1) describe the network power balance. The differential equations $f(\mathrm{x}, \mathrm{y}$, $\mathrm{z}$ ) in (1) describe the transient process of synchronous generators. The discrete equations $h(\mathrm{x}, \mathrm{y}, \mathrm{z})$ in (1) describe the trigger logic of equipment relays. The details of (1) can be found in Ref [4], and are omitted here due to the space limitation.

\section{Impact of utility-scale and distributed renewable energy on cascading failures}

Since the dynamics of renewable energy are significantly different from that of conventional units, it is necessary to characterize the impact of renewable energy on system dynamics in cascading failures for accurate assessment of blackout risk. Two key problems should be addressed to capture the impacts of renewable energy on cascading failure:

On the one hand, the dynamic model of renewable energy is required for stability analysis of large power grids. Utility-scale renewable energy has a large installed capacity and is directly connected to the transmission system. It has interactions with conventional units and transmission components. Thus, a dynamic model is required for utility-scale renewable energy [8]. However, the distributed renewable energy has a small capacity and is connected to the distribution network, so the cascading failure of transmission system generally does not consider its dynamics. This paper considers the impact of distributed renewable energy by the net load level at buses of the transmission system.

On the other hand, the relays for renewable energy also are required. Utility-scale renewable energy may be disconnected from the grid due to voltage or frequency disturbances. Similarly, the distributed renewable energy may also be disconnected under the disturbance of transmission system.

\section{Dynamic model for utility-scale renewable energy in cascading failures}

The dynamic models of renewable generation include detailed models and general models. The detailed model is generally designed for a specific unit type, which is mostly used to analyze the electromagnetic transient of a renewable energy generator under a grid disturbance. Although the detailed model can accurately reflect the internal dynamics of the generator, the model parameters are difficult to obtain and the modeling is complicated. If there are a large number of renewable energy generators or system faults to be simulated, the computational complexity is rather high. The general model is mainly designed for the dynamic simulation in the large power grid, which focuses on the dynamic interactions between the renewable energy plant and the transmission grid. The module in the general model can be used in both wind farms and photovoltaic power plants, which have good portability. Considering the analysis of cascading failures, this paper adopts a general model to describe the dynamics of utility-scale renewable energy.

The second-generation WECC renewable energy general dynamic model is widely used [8]. It includes 7 modules shown in Fig. 1.

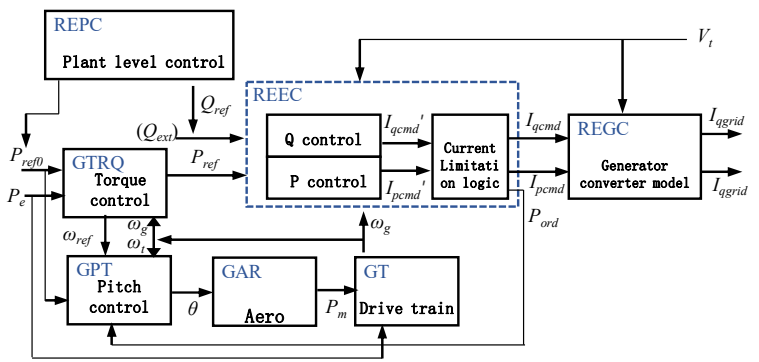

Fig.1 The second-generation WECC generic dynamic model for renewable generators

This paper uses the doubly-fed wind turbine to introduce the dynamic model of utility-scale renewable energy. Furthermore, this paper considers the secondgeneration WECC renewable energy generic model based on the following two premises.

1) The time scale for dynamics in cascading failures ranges from seconds to minutes. Generally, the millisecond power oscillation of the generator is not concerned, so the dynamic logic that has a time constant less than $0.02 \mathrm{~s}$ is removed and only its gain coefficient is considered. This can improve the efficiency of dynamic simulation in cascading failures.

2) Since the verification of the REPC module is still in progress, there is a lack of valid parameter settings [9]. Moreover, REPC corresponds to the minute-level plantlevel control system, and its priority is lower than the remaining two second-level electrical modules REEC and REGC. Therefore, this paper uses the REEC and REGC to describe the electrical dynamics of utility-scale renewable energy in cascading failures.

Based on the above premise, the dynamic equation of utility-scale renewable energy in cascading failures is established according to the WECC general model, with 
which the dynamic model of the power system with renewable energy can be constructed for cascading failures as (1).

The electrical module of the utility-scale renewable energy general model includes two parts: the converter control model (REEC module) and the renewable energy generator/converter model (REGC module). The corresponding dynamic equations are constructed as follows.

The REEC module describes active/reactive power control strategies under normal conditions and voltage disturbances. For normal conditions, REEC includes different control modes of reactive power such as constant power and constant voltage. This paper adopts the constant power control mode. When the voltage disturbance occurs, the reactive power is mainly dominated by the fault control logic. The considered REEC module is shown in Fig. 2. The input is the voltage $V_{t}$ at the point of integration, the active reference $P_{r e f}$, and the generator speed $\omega_{g}$. The output is the active current command $I_{p c m d}$ and the reactive current command $I_{q c m d}$. The corresponding control equations are constructed as follows:

$$
\begin{array}{r}
I_{q i n j}=\left\{\begin{array}{rr}
K_{q v}\left(V_{r e f 0}-V_{t}\right) & V_{t}<V_{d i p} \text { or } V_{t}>V_{u p} \\
0 & V_{d i p} \leq V_{t} \leq V_{u p}
\end{array}\right. \\
I_{q c m d}=\operatorname{sigm}\left(I_{q i n j}+\frac{Q_{e x t}}{V_{t}}\right) \\
P_{\text {ord }}=\operatorname{sigm}\left(\omega_{g} P_{r e f}\right) \\
I_{p c m d}=\min \left(\operatorname{sigm}\left(\frac{P_{\text {ord }}}{V_{t}}\right), \sqrt{I_{\max }^{2}-I_{q c m d}^{2}}\right)
\end{array}
$$

In the above model, (2) describes the reactive power support strategy of utility-scale renewable energy under abnormal voltage disturbance. (3) describes that the reactive current command $I_{q c m d}$ is jointly determined by normal operation control and abnormal operation control. Equations (4) and (5) describe that the active current command $I_{p c m d}$ is determined by the active power reference $P_{\text {ref }}$ and the current limit logic. The current limit logic considers the reactive current priority. The function $\operatorname{sigm}(x)$ is to use a sigmoid curve to mimic the limitation function of the controller [4]. The saturation region of the sigmoid curve can approximate the limiting effect while ensuring that the value of the differential equation is still differentiable when the limiting logic is in effect.

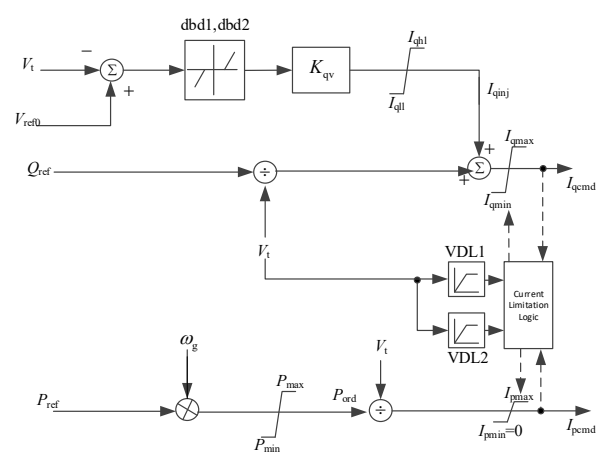

Fig.2 REEC control module

The control logic of the REGC module is shown in Fig. 3. It is used to describe the reactive power control of the converter under high voltage disturbance and the active power control under low voltage disturbance. The input is the active current command $I_{p c m d}$ and the reactive current command $I_{q c m d}$. The active current $I_{p g r i d}$ and the reactive current $I_{\text {qgrid }}$ injected into the grid by the network point correspond to equations (6) and (7), respectively.

$$
\begin{gathered}
I_{\text {pgrid }}=\left\{\begin{array}{lc}
0 & V_{t} \leq L p_{0} \\
\frac{V_{t}-L p_{0}}{L p_{1}-L p_{0}} I_{p c m d} & L p_{0}<V_{t} \leq L p_{1} \\
I_{p c m d} & V_{t}>L p_{1}
\end{array}\right. \\
I_{\text {qgrid }}=\left\{\begin{array}{lc}
I_{q c m d} & V_{t} \leq V_{o l i m} \\
I_{q c m d}-K_{h v}\left(V_{t}-V_{o l i m}\right) & V_{t}>V_{o l i m}
\end{array}\right.
\end{gathered}
$$

In the above model, equation (6) describes that when the bus voltage is low, the active current $I_{p g r i d}$ injected into the grid is reduced according to the voltage drop, which helps to stabilize the system. Equation (7) describes that when the bus voltage is higher than $V_{\text {olim }}$, the reactive power injection current $I_{\text {qgrid }}$ at the gridconnected point is adjusted according to the voltage deviation, which helps the voltage at the grid-connected point to be reduced to a normal level.

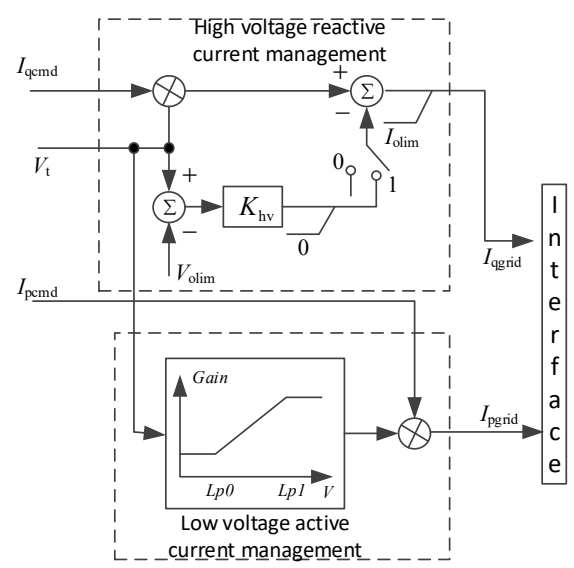

Fig. 3 REGC control module

The dynamic equations are also constructed for other modules including GTRQ, GPT, GAR, and GT. However, it is omitted here due to the space limitation. 


\section{Dynamic model for utility-scale renewable energy in cascading failures}

The propagation process of cascading failures mainly corresponds to the successive triggering of various equipment relays, which is described by the discrete equations in (1). Specifically, after solving the algebraic differential equations, the operating state of the system can be obtained. By comparing the system state with the logic of the relay, the relay triggering can be simulated. The logic of various relays is mainly described by the voltage, current, or frequency of the system. The voltage and current are determined by solving the power flow equations, and the frequency information is determined by the speed of the synchronous generators with close electrical distances [4], and the speed of the synchronous machines can be simulated by electromechanical transient equations.

In the cascading failure of a power system with renewable energy, it is necessary to consider the relay logic of utility-scale and distributed renewable energy, which is introduced as follows.

\subsection{Relays for utility-scale renewable energy}

The second-generation WECC renewable energy general model does not include a module for the voltage relay or frequency relay, so it is necessary to further establish a utility-scale renewable energy relay model. In this paper, it is considered that utility-scale renewable energy should satisfy the tolerance requirements for voltage disturbance [10]. When its terminal voltage $V_{t}$ is between 0.90 p.u. and 1.10 p.u., the utility-scale renewable generation should keep the connection with the grid. Otherwise, the time delay of the voltage relay is considered as:

$$
t_{\text {delay }}=\left\{\begin{array}{l}
0 s \quad V_{t} \leq 0.20 \text { or } V_{t} \geq 1.10 \\
\frac{V_{t}-0.20}{0.90-0.20} 0.625 s \quad 0.20<V_{t} \leq 0.90
\end{array}\right.
$$

Similarly, according to the frequency requirements [10], this paper considers that the threshold of the highfrequency relay of utility-scale renewable energy is 51 $\mathrm{Hz}$ and the delay is $0.2 \mathrm{~s}$, while the threshold of lowfrequency relay is $48 \mathrm{~Hz}$ and the delay is $0.2 \mathrm{~s}$.

\subsection{Relays for distributed renewable energy}

In order to prevent distributed renewable energy from forming islands and endangering the safety of the distribution network, anti-islanding relay is widely used [6]. When the operating status of the transmission system changes rapidly due to disturbances, distributed renewable energy may also be tripped by the antiislanding relay. For example, during the 8.9 blackout in the United Kingdom, $500 \mathrm{MW}$ of distributed renewable energy sources were disconnected from the grid, most of which were disconnected by RoCoF relay [1]. For this reason, this paper considers RoCoF relay as the anti- islanding relay, for which the threshold is $0.50 \mathrm{~Hz} / \mathrm{s}$ and the delay is $0.1 \mathrm{~s}$ [6].

\subsection{Other relays}

In order to protect the safety of conventional generators, the conventional generators need to be removed from the system when the voltage/frequency deviation is too large. The threshold of high-frequency relay considered in this paper is $51.5 \mathrm{~Hz}$, and the delay is $10.0 \mathrm{~s}$. The action threshold of low-frequency relay is $47.5 \mathrm{~Hz}$, and the action delay is $10.0 \mathrm{~s}$. And the threshold for the overvoltage relay is $1.3 \mathrm{p}$.u. while the delay is $1.0 \mathrm{~s}$. The threshold for under-voltage relay is $0.8 \mathrm{p}$.u. and the delay is $1.0 \mathrm{~s}$.

In addition, the relay settings for transmission lines, UFLS, and UVLS, are similar to those in [4].

\section{Dynamic cascading failure model for power system with renewable energy}

With the dynamic equations and relay logic, the dynamic propagation process of cascading failures in power systems with renewable energy can be simulated as follows:

Step 1) System state initialization. The AC power flow is solved, and the state variables of the differential equation system are initialized according to the power flow calculation [11]. Besides, initialize the current time $t$ to $0 \mathrm{~s}$ and the dynamic simulation step size $d t$ to $0.01 \mathrm{~s}$, respectively.

Step 2) Set the initial fault as the outages of transmission lines.

Step 3) Check the connection of power system. If the system has islands, the data of each subsystem is generated, and the follow-up process of the cascading failure is simulated in each subsystem.

Step 4) Update the admittance matrix of the current system, and then solve the algebraic equations.

Step 5) Solve algebraic differential equations using the implicit trapezoidal method [11]. If it converges, go to step 6. Otherwise, it is judged whether the step length $d t$ is greater than the minimum step length $d t_{\min }$. If it is, set $d t=0.5 \mathrm{dt}$ and solve again. Otherwise, the current subsystem becomes unstable, and all loads are removed. The current subsystem simulation ends.

Step 6) According to the system operating status in Step 5 and component relay logic, update the relay status.

Step 7) Update the current time $t=t+d t$. Check whether a fault clearing occurs or the relay action delay is 0 . If it exists, update the component status and go to step 3.

Step 8) Increase the simulation step size to $d t=2 d t$ to accelerate the simulation when no new discrete events occur [4].

Step 9) If $t$ is greater than the preset simulation time $t_{\text {max }}$, go to step 10, otherwise go to step 5 .

Step 10) The cascading failure simulation ends. 


\section{CASE STUDIES}

This section verifies the proposed model on the IEEE 39-bus system. The original data of the system includes 10 generators and 46 transmission lines. The topology is shown in Fig. 4. The electrical parameters of each component and the controller parameters of the conventional unit are detailed in [4]. The proposed dynamic cascading failure model is coded on MATLAB.

The two conventional units at buses 30 and 31 in the original IEEE 39-bus system are replaced with wind farms that have equal capacity. The wind power penetration is about $20 \%$. The wind farm model adopts the doubly-fed wind turbines, for which the WECC general model parameters are detailed in [9]. Besides, the output of distributed renewable energy at each load bus is set as $10 \%$ of the original load, namely, the net load of each bus is $90 \%$ of the original load. The load adopts a constant impedance model. The dynamic simulation time $t_{\max }$ is set as $100 \mathrm{~s}$.

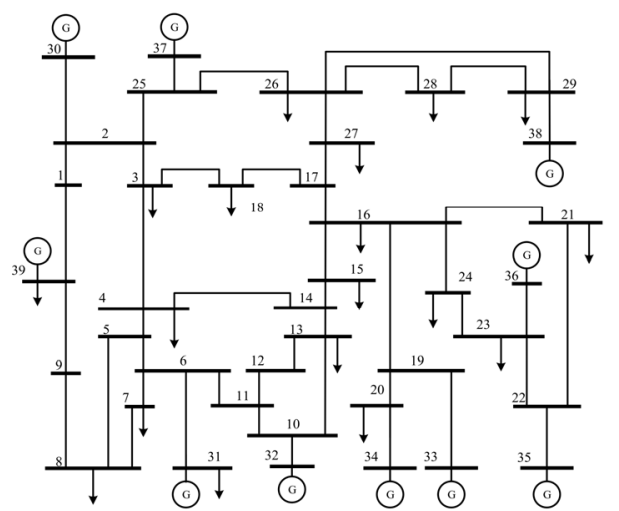

Fig.4 Single-line diagram of the IEEE 39-bus system

The following gives an example of the dynamic simulation process of a cascading failure by setting the initial fault as the disconnection of branches 2-25 and 631 at $\mathrm{t}=0.1 \mathrm{~s}$. The cascading fault propagation process is shown in Table 1, and the corresponding voltage dynamics and frequency dynamics are shown in Fig. 5 and Fig. 6, respectively.

Table 1. Cascading failures induced by the outages of branches 2-25 and 6-31.

\begin{tabular}{|c|c|}
\hline Time & Events \\
\hline $0.10 \mathrm{~s}$ & Branches 2-25 and 6-31 are disconnected \\
\hline $0.20 \mathrm{~s}-2.25 \mathrm{~s}$ & $\begin{array}{l}\text { The system frequency drops, and the RoCoF } \\
\text { relays of distributed renewable energy } \\
\text { sources at buses } 25,39 \text {, and } 27 \text { are tripped. }\end{array}$ \\
\hline $2.76 \mathrm{~s}$ & Overcurrent relay of branch $16-17$ is tripped \\
\hline $3.44 \mathrm{~s}$ & Overcurrent relay of branch 4-14 is tripped \\
\hline $3.52 \mathrm{~s}-3.66 \mathrm{~s}$ & $\begin{array}{c}\text { The low-voltage load shedding of buses } 7,8 \\
\text { and } 4 \text {, is tripped in sequence }\end{array}$ \\
\hline $3.92 \mathrm{~s}$ & $\begin{array}{l}\text { The overcurrent relay of branch } 3-18 \text { is } \\
\text { tripped, and the system is separated }\end{array}$ \\
\hline $4.36 \mathrm{~s}$ & $\begin{array}{l}\text { The low-frequency relay of the wind farm at } \\
\text { bus } 30 \text { is tripped }\end{array}$ \\
\hline $4.78 \mathrm{~s}$ & $\begin{array}{c}\text { Low frequency load shedding at buses } 3,4 \text {, } \\
7,8 \text {, and } 39 \text { is tripped }\end{array}$ \\
\hline $14.4 \mathrm{~s}$ & $\begin{array}{l}\text { The conventional unit at bus } 39 \text { is tripped } \\
\text { due to the low frequency relay }\end{array}$ \\
\hline
\end{tabular}

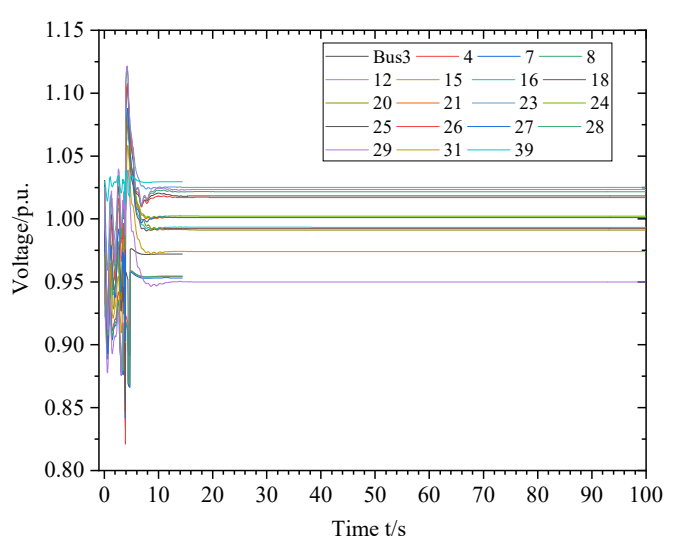

Fig.5 Voltage curves of load buses after the outages of transmission lines 2-25 and 6-31

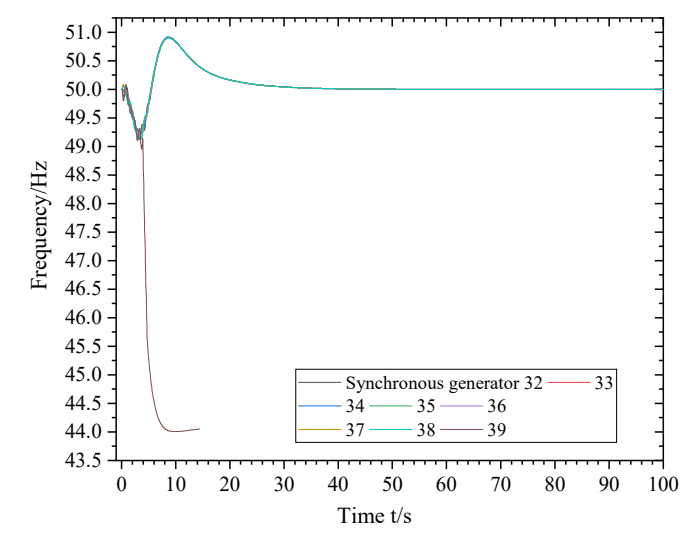

Fig.6 Frequency curves of conventional generators after the outages of transmission lines 2-25 and 6-31

As shown in Table 1, the disconnection of branch 631 at $0.1 \mathrm{~s}$ causes the wind farm at bus 31 to be isolated, leading to an active power shortage of 515.61 MW. Because the generator output is lower than the load demand, the frequencies of some conventional units drop. During the period from $0.1 \mathrm{~s}$ to $0.2 \mathrm{~s}$, the RoCoF at bus 39 and bus 32 are $0.91 \mathrm{~Hz} / \mathrm{s}$ and $0.52 \mathrm{~Hz} / \mathrm{s}$, respectively, which exceed the threshold of the RoCoF relay of distributed renewable energy $(0.50 \mathrm{~Hz} / \mathrm{s})$. Therefore, the distributed renewable energy at buses 25 and 39 is tripped firstly. The trip of distributed renewable energy sources further increases the active power shortage and accelerates the rate of decrease of frequency. During the period from $0.20 \mathrm{~s}$ to $2.25 \mathrm{~s}$, the RoCoF relays of other sixteen buses, such as buses 18 and 27, are tripped in sequence. Due to the increase of the net load, branch 1617 becomes overloading and is tripped at $2.76 \mathrm{~s}$ due to the overcurrent relay action. Subsequently, branch 4-14 also becomes overloading due to the power flow transfer and is tripped at $3.44 \mathrm{~s}$. Due to the topology change of the transmission system, the voltages of buses 7,8 , and 4 fluctuate between $0.87 \mathrm{pu}$ and $0.89 \mathrm{pu}$, which is lower than $0.90 \mathrm{pu}$, and thus the corresponding low-voltage load shedding is tripped during $3.52 \mathrm{~s}$ to $3.66 \mathrm{~s}$. The change of the power flow cause the trip of the overcurrent relay of branch 3-18 at $3.92 \mathrm{~s}$, and then the system was disconnected again.

At $3.92 \mathrm{~s}$, the system separates into two subsystems. Subsystem 1 includes buses 1, 2, 3, 4, 5, 6, 7, 8, 9, 30, 
and 39. Since the power supply of subsystem 1 only includes the conventional generator at bus 39 and the wind farm at bus 30 , the load demand (2367.81 MW) is much greater than the generated power (1169.78 MW), so the frequency of the conventional unit at bus 39 drops rapidly. At $4.36 \mathrm{~s}$, the wind farm at bus 30 is disconnected due to the low-frequency relay action, and then at $4.78 \mathrm{~s}$, the low-frequency load shedding at buses $3,4,7,8$, and 39 are tripped. At $14.4 \mathrm{~s}$, the conventional unit at bus 39 is tripped by the low-frequency relay delay. At this time, all the load in subsystem 1 is out of service. Besides, the load demand in subsystem 2 is lower than the power generation, and the frequency of the conventional unit has a temporary rise and then returns to the normal range under the control of the governor.

It can be seen from the above process that the integration of utility-scale and distributed renewable energy sources has changed the power regulation ability and disturbance tolerance. Therefore, during cascading failures, the risk of generator disconnection increases, and the power transmission components are also subject to interaction influences.

\section{Conclusions}

This paper considers the impact of utility-scale and distributed renewable energy on the dynamics of the power system, and proposes a dynamic model of cascading failures for the power system with renewable energy. The proposed cascading failure model is illustrated with the IEEE 39-bus system, and the influence of utility-scale and distributed renewable energy on the cascading failure risk is analyzed. Compared with the cascading failure model based on steady-state analysis, the proposed dynamic model can analyze the propagation mechanism of cascading failures more comprehensively, and provide an effective reference for operators to quantify the risk of blackouts.

\section{References}

1. Australian Energy Market Operator (AEMO). Black System South Australia 28 September 2016[R/OL]

2. NGESO. Technical Report on the events of 9 August 2019[R/OL]

3. M.H. Athari, Z. Wang. Impacts of wind power uncertainty on grid vulnerability to cascading overload failures. IEEE Trans. Sustain. Energy, 9, 128-137 (2018)

4. J. Song, E. Cotilla-Sanchez, G. Ghanavati, et al. Dynamic modeling of cascading failure in power systems. IEEE Trans. Power Syst., 31, 2085-2095 (2016)

5. X. Zhang, Y. Zhong, S. Mei, et al. Blackout risk analysis of power system integrated with DFIG wind farm. Adv. Tech. of Elec. Eng. and Energy, 35, 1-7 (2016)

6. T. Amraee, M. Darebaghi, A. Soroudi, et al. Probabilistic under frequency load shedding considering RoCoF relays of distributed generators. IEEE Trans. Power Syst., 33, 3587-3598 (2018)

7. K. Yamashita, H. Renner, S. Villanueva, et al. Industrial recommendation of modeling of inverterbased generators for power system dynamic studies with focus on photovoltaic. IEEE Power Energy Technol. Syst. J., 5, 1-10 (2018)

8. P. Pourbeik. Specification of the second generation generic models for wind turbine generators. USA: EPRI, (2013)

9. G. Lammert, L.D.P. Ospina, P. Pourbeik, et al. Implementation and validation of WECC generic photovoltaic system models in DIgSILENT PowerFactory. 2016 IEEE Power and Energy Society General Meeting (PESGM). 1-6, (2016)

10. Rodrigues E M G, Osório G J, Godina R, et al. Grid code reinforcements for deeper renewable generation in insular energy systems $[\mathrm{J}]$. Renew. Sust. Energ. Re., 53, 163-177 (2016)

11. X. Wang, Y. Song, M. Irving. Modern power systems analysis[M]. Springer Science \& Business Media, 2010. 